\title{
IPHAS extinction distances to planetary nebulae
}

\author{
C. Giammanco ${ }^{1,12}$, S. E. Sale ${ }^{2}$, R. L. M. Corradi ${ }^{1,12}$, M. J. Barlow ${ }^{3}$, K. Viironen ${ }^{1,13,14}$, L. Sabin ${ }^{4}$, \\ M. Santander-García ${ }^{5,1,12}$, D. J. Frew ${ }^{6}$, R. Greimel ${ }^{7}$, B. Miszalski ${ }^{10}$, S. Phillipps ${ }^{8}$, A. A. Zijlstra9 ${ }^{9}$, \\ A. Mampaso ${ }^{1,12}$, J. E. Drew ${ }^{2,10}$, Q. A. Parker ${ }^{6,11}$, and R. Napiwotzki ${ }^{10}$ \\ 1 Instituto de Astrofísica de Canarias (IAC), C/ vía Láctea s/n, 38200 La Laguna, Spain \\ e-mail: corrado@iac.es \\ 2 Astrophysics Group, Imperial College London, Blackett Laboratory, Prince Consort Road, London SW7 2AZ, UK \\ 3 Department of Physics and Astronomy, University College London, Gower Street, London WC1E 6BT, UK \\ 4 Instituto de Astronomía, Universidad Nacional Autónoma de México, Apdo. Postal 877, 22800 Ensenada, B.C., Mexico \\ 5 Isaac Newton Group of Telescopes, Ap. de Correos 321, 38700 Sta. Cruz de la Palma, Spain \\ ${ }^{6}$ Department of Physics, Macquarie University, NSW 2109, Australia \\ 7 Institut für Physik, Karl-Franzens Universität Graz, Universitätsplatz 5, 8010 Graz, Austria \\ 8 Astrophysics Group, Department of Physics, Bristol University, Tyndall Avenue, Bristol BS8 1TL, UK \\ 9 Jodrell Bank Centre for Astrophysics, School of Physics and Astronomy, University of Manchester, Oxford Road, \\ M13 9PL Manchester, UK \\ ${ }^{10}$ Centre for Astrophysics Research, STRI, University of Hertfordshire, College Lane Campus, Hatfield AL10 9AB, UK \\ 11 Anglo-Australian Observatory, PO Box 296, Epping, NSW 1710, Australia \\ 12 Departamento de Astrofísica, Universidad de La Laguna, 38205 La Laguna, Tenerife, Spain \\ 13 Centro Astronómico Hispano Alemán, Calar Alto, C/Jesús Durbán Remón 2-2, 04004 Almeria, Spain \\ 14 Centro de Estudios de Física del Cosmos de Aragón (CEFCA), C/General Pizarro 1-1, 44001 Teruel, Spain
}

Received 19 March 2010 / Accepted 3 July 2010

\begin{abstract}
Aims. The determination of reliable distances to planetary nebulae (PNe) is a major difficulty in the study of this class of objects in the Galaxy. The availability of new photometric surveys such as IPHAS (the INT/WFC photometric $\mathrm{H}_{\alpha}$ survey of the northern Galactic plane) covering large portions of the sky provide an opportunity to apply the so-called extinction method to determine the distances of a large number of objects.

Methods. The technique is applied to a sample of $137 \mathrm{PNe}$ located between -5 and 5 degrees in Galactic latitude, and between 29.52 and 215.49 degrees in longitude. The characteristics of the distance-extinction method and the main sources of errors are carefully discussed.

Results. The data on the extinction of the PNe available in the literature, complemented by new observations, allow us to determine extinction distances for $70 \mathrm{PNe}$. A comparison with statistical distance scales from different authors is presented.
\end{abstract}

Key words. catalogs - planetary nebulae: general

\section{Introduction}

The measurements of the distances to Galactic planetary nebulae $(\mathrm{PNe})$ has been a longstanding difficulty. Obtaining an accurate distance scale for Galactic PNe will allow us to infer the total number of PNe in the Galaxy, which has important implications for the Galactic ultraviolet radiation field, the total processed mass returned to the interstellar medium, and more generally to our understanding of the chemical evolution of the Galaxy.

A method that is a priori independent of assumptions about the physical or geometrical properties of the nebulae is the extinction method. Assuming that the interstellar extinction to a certain nebula can be determined, if one is able to build up the extinction-distance relation using field stars around the line of sight to the nebula, the relation can be used to infer the distance to the planetary nebula (PN).

The application of this method is not new. Lutz (1973) measured the distance to 6 PNe using some 10 field stars per object. The method was later applied by Acker (1978) who provided reliable distance values for $11 \mathrm{PNe}$ and a rough estimate for
34 other ones, and by Gathier et al. (1986) who measured the distance to 12 PNe using some 50 stars per PN. In the last paper, a comprehensive discussion of the different aspects of this method can be found. The number of stars used to determine distances was increased by Pollacco \& Ramsay (1992) who, using a colour analysis of the field stars, were able to perform an accurate spectral classification for stars later than F5 type. As late-type stars constitute the most numerous objects in all Galactic-plane directions, they allow an extensive application of this method to determine distances to PNe in the Galaxy.

The availability of the IPHAS $\mathrm{H} \alpha$ survey of the northern Galactic plane (Drew et al. 2005) and its forthcoming extension to the south (VPHAS+) opens new doors for the application of the method. IPHAS allows us to determine extinction-distance curves using a large number of field stars, typically several hundred in areas as small as $10^{\prime} \times 10^{\prime}$ around each line of sight. The technique is presented and discussed by Sale et al. (2009). In this paper, we present its application to Galactic PNe. We determine the distances to $70 \mathrm{PNe}$ included in the ESO/Strasbourg catalogue (Acker et al. 1992), and compare the distances obtained 
with those obtained by other authors using different methods. This shows how IPHAS and its successor surveys potentially provide a new and powerful tool to obtain distances to a large number of Galactic PNe.

\section{The data}

IPHAS is a wide-field, CCD, $\mathrm{H} \alpha$ survey of the northern Galactic plane (Drew et al. 2005), which was carried out at the $2.5 \mathrm{~m}$ Isaac Newton Telescope on La Palma, Spain. Imaging was also performed in the $r^{\prime}$ and $i^{\prime}$ bands down to $r^{\prime} \sim 20(10 \sigma)$. The high quality photometry and characteristics of the survey permit the spectral classification of main-sequence stars to be determined, based on the $\mathrm{H} \alpha$ line strength. The availability of reliably calibrated $\left(r^{\prime}-\mathrm{H}_{\alpha}\right)$ colours permit the spectral classification of most stars, while the $\left(r^{\prime}-i^{\prime}\right)$ colour provides their extinctions, allowing distances to be estimated from the $r^{\prime}$ measurements. The limiting magnitude in $r^{\prime}$ permits extinctions to be measured to distances of up $\sim 10 \mathrm{kpc}$.

IPHAS covers the Galactic latitude range between -5 and $5 \mathrm{deg}$, and longitude range between 29.52 and 215.49. In this region of the sky, there are 190 known PNe according to the ESO/Strasbourg catalogue (Acker et al. 1992). We were able to extract a reddening for 137 of them from different sources; for $27 \mathrm{PNe}$, we present the first determinations.

\subsection{PN extinctions extracted from the literature}

The ESO/Strasbourg catalog provides the $\mathrm{H}_{\alpha} / \mathrm{H}_{\beta}$ line ratio for most of the PNe considered here. These data were measured from spectra obtained using two different telescopes: the $1.52 \mathrm{~m}$ ESO telescope for the Southern nebulae, and the $1.93 \mathrm{~m}$ OHP telescope for the Northern ones. More information about the instruments (photographic plate or CCD) and spectra can be found in Acker et al. (1992, SECGPN), Acker \& Stenholm (1987), and Stenholm \& Acker (1987). Tylenda et al. (1992) used these SECGPN line intensities to obtain the value of the extinction constant roughly determined for about $900 \mathrm{PNe}$. In this paper, the visual extinction was obtained from the line ratios by applying the formulae

$c_{\beta}=2.84 \cdot \log \left(\frac{\left(\mathrm{H}_{\alpha} / \mathrm{H}_{\beta}\right)}{2.86}\right)$,

$A_{\mathrm{v}}=2.15 \cdot c_{\beta}$

which were taken from Fitzpatrick (2004). Here we assumed $R=3.1$, to be consistent with the method by which the IPHAS extinction curves are built (see Sect. 3).

Other compilations from which $c_{\beta}$ was retrieved are Cahn et al. (1992) and in a few cases Stasińska et al. (1992). The latter paper compared the optical and radio extinction determinations, using the same "SECGPN" line fluxes as Tylenda et al. (1992), and adding some other measurements, for a total of $130 \mathrm{PNe}$. For individual nebulae, these listings where supplemented with data from other papers, where available. In all cases, when the assumed reddening law is reported in a paper, we normalize the published extinction using Eqs. (1) and (2). This is specifically, the case for data in Cahn et al. (1992) and Stasińska et al. (1992). For most of the nebulae, the only available extinction coefficient comes from the Strasbourg catalog, while for a subset there are multiple determinations. For a small number of nebulae, there is a very extensive literature, and in those cases, we selected what we understood to be the most accurate determination of $c_{\beta}$ after

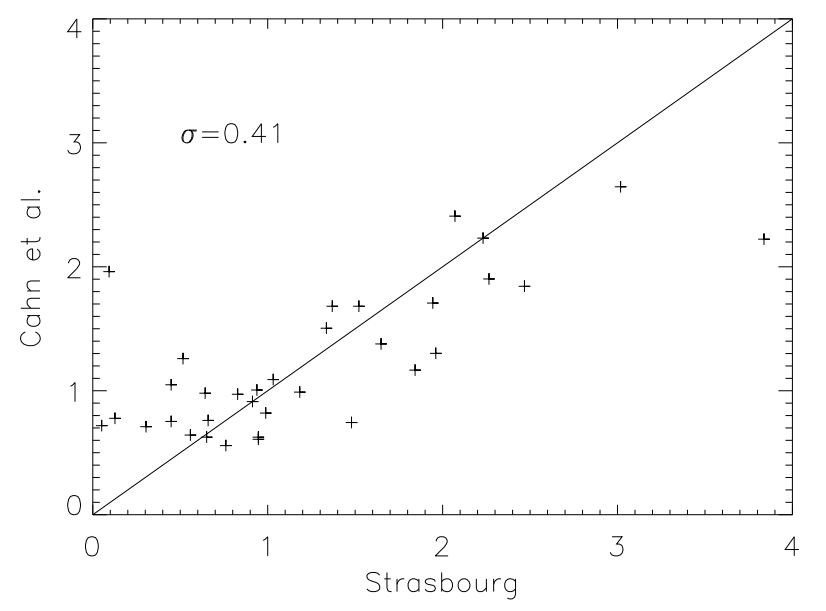

Fig. 1. $c_{\beta}$ measurements by by Cahn et al. (1992) plotted against data in Acker et al. (1992). The dispersion is around 0.4 in $c_{\beta}$.

a careful analysis of bibliographic sources. In addition, for some nebulae we also present new optical $c_{\beta}$ determinations.

A general rule, in the case of multiple determinations and the absence of other information (the quality of one measurement with respect to another), is to choose the smallest $c_{\beta}$. The main reason is that slit spectra taken at non-negligible zenith distances are often affected by differential atmospheric dispersion which removes blue light leading to an overestimate of the $c_{\beta}$ 's. Extinction measurements obtained from radio data are free from this effect, but have values that are systematically lower than those determined from the Balmer decrement (Stasińska et al. 1992; Ruffle et al. 2004). To ensure that we have a homogeneous set of measurements, we only consider in this paper $c_{\beta}$ obtained from optical measurements.

Finally, to illustrate the differences between extinction determinations by different authors, we show in Fig. 1 the $c_{\beta}$ values obtained by Cahn et al. (1992) versus those computed from fluxes in the ESO/Strasbourg catalog. The associated scatter is $\sigma=0.41$, which shows how critical the choice of the extinction values can be. We note that data from the ESO/Strasbourg catalog are not all of the same quality, those for the northern nebulae $(56<l<184)$ observed at the OHP being of generally lower quality than those of (mainly southern) nebulae observed at ESO.

\subsection{New extinction determinations}

Twenty-six PNe considered in this paper (see Tables 1 and 3) were observed at the $2.5 \mathrm{~m}$ Isaac Newton Telescope at the Observatorio del Roque de los Muchachos, from August 23 to 30, 1997. The IDS spectrograph was used with its R300V grating, which provides a reciprocal dispersion of $3.31 \AA$ per pixel of the $1 \mathrm{k} \times 1 \mathrm{k}$ Tek3 detector, and a spectral coverage from 3700 to $6900 \AA$. The slit width was 1.5 arcsec projected on the sky, providing a spectral resolution of $6.7 \AA$. Total exposure times on each nebula varied from a few seconds for the brightest target (NGC 7027) up to one hour for the faintest ones; exposures were split to ensure that both the bright and faint nebular lines were reliably detected without saturation. Several spectrophotometric standards were observed during each night to help perform a relative flux calibration. Data reduction was performed using the package onedspec in IRAF.

An additional two PNe (PC 20 and Sa 3-151) were observed with the Dual Beam Spectrograph (DBS, Rodgers et al. 1988) on 
Table 1. Distances inferred for the NGC sample.

\begin{tabular}{|c|c|c|c|c|c|c|c|c|c|c|}
\hline \multirow[t]{2}{*}{ PNG } & \multirow[t]{2}{*}{ Name } & \multicolumn{5}{|c|}{$A_{\mathrm{v}}$} & \multicolumn{4}{|c|}{ distance $[\mathrm{pc}]$} \\
\hline & & Stras. & Cahn & Stas. & this paper & Others & $\mathrm{dC}$ & dVst & $\mathrm{dMa}$ & this paper \\
\hline $033.8-02.6$ & NGC 6741 & 1.96 & 1.96 & 1.93 & & 1.73 & 2047 & 2260 & 1700 & $2680 \pm 110$ \\
\hline $041.8-02.9$ & NGC 6781 & & 2.09 & 1.96 & $2.16 \pm 0.11$ & 1.78 & 699 & 840 & 900 & $<1000$ \\
\hline $045.7-04.5$ & NGC 6804 & & 1.85 & & $1.85 \pm 0.11$ & 1.58 & 1709 & 1660 & 1600 & $<800$ \\
\hline $046.4-04.1$ & NGC 6803 & 0.96 & 1.62 & 1.60 & & 1.14 & 2987 & 3190 & 2500 & $950 \pm 130$ \\
\hline $060.8-03.6$ & NGC 6853 & & 0.36 & & & 0.55 & 262 & 400 & 400 & $<800$ \\
\hline $065.9+00.5$ & NGC 6842 & 0.96 & 2.04 & & $2.08 \pm 0.15$ & & 1366 & & 1700 & $2700 \pm 950 \dagger$ \\
\hline 069.4-02.6 & NGC 6894 & & 1.80 & & $1.85 \pm 0.11$ & 1.44 & 1653 & 2000 & 1500 & $1000 \pm 100$ \\
\hline $074.5+02.1$ & NGC 6881 & 3.27 & 3.62 & & & 4.12 & 2473 & 3950 & 1700 & n.d. \\
\hline 084.9-03.4 & NGC 7027 & & 2.53 & & $2.97 \pm 0.46$ & & 273 & 630 & 700 & n.d. \\
\hline $088.7-01.6$ & NGC 7048 & 0.65 & 1.53 & & $1.20 \pm 0.13$ & 1.27 & 1598 & 2220 & 1200 & $1000-2000 \dagger$ \\
\hline $089.0+00.3$ & NGC 7026 & 1.40 & 1.34 & & $2.10 \pm 0.11$ & 1.69 & 1902 & 1940 & 900 & $<1500$ \\
\hline $107.8+02.3$ & NGC 7354 & 2.94 & 3.62 & & $3.99 \pm 0.08$ & 3.23 & 1271 & 1230 & 800 & $1000 \pm 150$ \\
\hline
\end{tabular}

Notes. Columns labelled as "Stras", "Cahn", and "Stas" correspond to the Strasbourg catalog, Cahn et al. (1992), and Stasińska et al. (1992), as well as "dC" and "dVst" are taken from Cahn et al. (1992) and van de Steene \& Zijlstra (1994), respectively, while "dMa" from Maciel (1984). "Other" references are given in the text. When significantly different extinction estimates are available, the values reported for the distances are obtained using the absorption values in bold face. Errors are statistical originating in the linear fit to the distance extinction curve, except for NGC 6842 and NGC 7048, where we have also included the errors in the extinction measurement.

May 10 and 14, 2008 by B. Miszalski at the Australian National University 2.3-m telescope. The exposure times were $300 \mathrm{~s}$ and $150 \mathrm{~s}$, respectively to achieve a signal-to-noise ratio of about 20 in the peak intensity of $\mathrm{H}_{\beta}$. The $1200 \mathrm{~B}$ and $1200 \mathrm{R}$ gratings were used with a slit width of $2^{\prime \prime}$ (positioned at PA $=270 \mathrm{deg}$ ) to provide wavelength coverage windows of 4030-5050 $\AA$ and $6245-7250 \AA$ at a resolution of $\sim 1.6 \AA(F W H M)$. Data reduction was performed using IRAF and a number of flux standards were observed each night to derive the spectrophotometric response across the separate blue and red spectrograph arms.

\section{IPHAS extinction-reddening curves for the PNe}

Extinction-distance relationships for the lines of sight toward the sample of 136 Galactic PNe in the IPHAS area were computed with the algorithm MEAD, as described by Sale et al. (2009). MEAD exploits a feature of the IPHAS colour-colour plane, whereby it is possible to accurately determine simultaneously the spectral type of a star and its reddening, avoiding the problems caused by the significant degeneracies that affecting other filter systems and with only a slight dependence on a Galactic model.

Typically, several thousand A to K4 stars in a box of sides $10^{\prime}$, centred on the PNe, were employed in the computation of each relationship. Distances to each star were derived from their apparent magnitude, the MEAD extinction, and the absolute magnitude of the star derived from the MEAD spectral type. The photometric errors were propagated to determine an error in the estimated distance to each star. The stars were then binned by distance, with each bin being at least 100 pc deep, containing at least 8 stars and having a total signal-to-noise ratio in the bin of at least 130. In a small departure from the method described in Sale et al. (2009), the prior probabilities of each luminosity class were determined on a sightline by sightline basis. MEAD evaluates monochromatic extinctions, which can be subsequently converted to $A_{\mathrm{v}}$ using the $R=3.1$ reddening law of Fitzpatrick (1999).

\section{The inversion of distance-extinction curves}

The extinction-distance curves obtained are in general linear for a significant range of distances, until they generally flatten out
Table 2. List of PNe with a reported visual extinction more than 1 mag larger than the plateau of the corresponding distance-extinction curve.

\begin{tabular}{ccccc}
\hline \hline PNG & Name & $A_{\mathrm{v}}$ & $A_{\mathrm{v}}$ Plateau & Reference \\
\hline $032.0-03.0$ & K3-18 & 5.56 & 4.5 & 1 \\
$032.9-02.8$ & K3-19 & 4.49 & 3 & 1 \\
$048.1+01.1$ & K3-29 & 6.31 & 3.5 & 2 \\
$051.0+02.8$ & WhMe1 & 7.60 & 5 & 1 \\
$054.4-02.5$ & M1-72 & 4.80 & 2.5 & 1,3 \\
$055.2+02.8$ & He2-432 & 6.32 & 4 & 1 \\
$059.9+02.0$ & K3-39 & 5.78 & 3.8 & 1 \\
$064.9-02.1$ & K3-53 & 6.25 & 3.8 & 1 \\
$069.2+02.8$ & K3-49 & 4.76 & 3.4 & 1 \\
$072.1+00.1$ & K3-57 & 4.56 & 3 & 1 \\
$094.5-00.8$ & K3-83 & 4.72 & 3 & 1 \\
$104.1+01.0$ & B12-1 & 5.76 & 4 & 1 \\
$110.1+01.9$ & PM1-339 & 7.35 & 6 & 4 \\
$112.5+03.7$ & K3-88 & 5 & 4 & 1 \\
\hline
\end{tabular}

Notes. The principal source of the $\mathrm{H}_{\alpha} / \mathrm{H}_{\beta}$ ratio is the ESO/Strasbourg catalog marked as (1) in the column "Reference", while the other sources are: (2) Stasińska et al. 1992; (3) Cahn et al. 1992; and (4) Manchado et al. 1989. ${ }^{(\dagger)} \mathrm{K} 3-18$ is a possible symbiotic star.

and reach an asymptotic value once the line of sight leaves the dust layer within the Galactic plane (Fig. 2). Even in the cases where they show a more complex shape, it is generally possible to select a linear region around the point in which we are interested, e.g; the $A_{\mathrm{v}}$ measured for the nebula.

We assume that the measured $A_{\mathrm{v}, i}$ are distributed around a true value $A_{\mathrm{v}, i}^{t}=a+b d_{i}$ following a Gaussian law whose dispersion is $\sigma_{A}$. If so, a least square fit to the selected part of the curve allows us to determine all the needed parameters. Around a point, for each $d$, the probability-distribution in particular that measures a given $A_{\mathrm{v}}$ is $P\left(A_{\mathrm{v}} \mid d\right)$. The next step is to invert this distribution, i.e.; to obtain the probability of finding a distance $d$ for a given $A_{\mathrm{v}, i}: P\left(d \mid A_{\mathrm{v}, i}\right)$. We note that

$P\left(d \mid A_{\mathrm{v}, i}\right)=\frac{P\left(A_{\mathrm{v}, i} \mid d\right)}{\int P\left(A_{\mathrm{v}} \mid d\right) \mathrm{d} A_{\mathrm{v}}}$

and using the previous assumptions of linearity and a Gaussian distribution for $P\left(A_{\mathrm{v}} \mid d\right)$, the new distribution for $P\left(d \mid A_{\mathrm{v}}\right)$ is itself 
Table 3. Distance to the whole sample of PNe.

\begin{tabular}{|c|c|c|c|c|c|c|c|c|c|c|c|}
\hline \multirow[t]{2}{*}{ PNG } & \multirow[t]{2}{*}{ Name } & \multicolumn{5}{|c|}{$c_{\beta}$} & \multicolumn{4}{|c|}{ Distance $[\mathrm{pc}]$} & \multirow[t]{2}{*}{ Reference } \\
\hline & & $\begin{array}{l}\text { Stras. } \\
\text { (1) }\end{array}$ & $\begin{array}{l}\text { Cahn } \\
(2)\end{array}$ & $\begin{array}{l}\text { Stas. } \\
(3)\end{array}$ & $\begin{array}{l}\text { this } p \text {. } \\
\text { (4) }\end{array}$ & $\begin{array}{l}\text { Other } \\
(5)\end{array}$ & Cahn & Vst. & Ma. & $\begin{array}{c}\text { THIS } \\
\text { PAPER }\end{array}$ & \\
\hline $031.7+01.7$ & PC20 & 2.51 & & & 3.04 & & & & & $>1100$ & \\
\hline $032.5-03.2$ & K3-20 & 1.88 & & & & & & & & $>1100$ & \\
\hline $032.7-02.0$ & M1-66 & 1.39 & & & 1.44 & & 3439 & 5000 & & $>3000$ & \\
\hline 033.2-01.9 & Sa3-151 & & & & 1.52 & & & & & $3500 \pm 300$ & \\
\hline 035.9-01.1 & Sh2-71 & 1.65 & 1.38 & & 1.18 & & 997 & & & $<1000$ & \\
\hline $038.7-03.3$ & M1-69 & 1.32 & & & & & & & & $3300 \pm 500$ & \\
\hline 040.4-03.1 & K3-30 & 1.67 & & 1.66 & & & 6291 & 6550 & & $>3000$ & \\
\hline $041.2-00.6$ & HaTr14 & 0.56 & & & & & & & & $<1000$ & \\
\hline $041.8+04.4$ & K3-15 & 1.28 & & & & & & & & $>6000$ & \\
\hline $043.0-03.0$ & M4-14 & 1.42 & & & & & 6692 & 7570 & 1600 & $2600 \pm 300$ & \\
\hline $043.1+03.8$ & M1-65 & 1.24 & & 1.23 & & & 6536 & 6280 & & $3200 \pm 900$ & \\
\hline $043.3+02.2$ & PM1-276 & & & & & 1.74 & & & & $1350 \pm 100$ & 1 \\
\hline $045.4-02.7$ & Vy2-2 & 1.94 & 1.71 & & & & 2159 & & & $2300 \pm 170$ & \\
\hline $046.3-03.1$ & PB9 & 1.81 & & & & & 4661 & 3990 & & $3800 \pm 450$ & \\
\hline $047.1+04.1$ & K3-21 & 1.15 & & & & & & & 10500 & $2700 \pm 366$ & \\
\hline 047.1-04.2 & A62 & & 2.07 & & & 0.18 & 494 & & 1100 & $<900$ & 2 \\
\hline $048.0-02.3$ & PB10 & 2.01 & & & & & 4685 & 3830 & & $>2000$ & \\
\hline $048.5+04.2$ & K4-16 & 1.51 & & 1.50 & & & 15127 & & & $>5000$ & \\
\hline $048.7+01.9$ & He2-429 & 2.12 & & & & 1.87 & 3383 & & & $1500 \pm 600$ & 3 \\
\hline $049.4+02.4$ & He2-428 & 1.23 & & & & 1.14 & & & & $1000 \pm 100$ & 7 \\
\hline $050.1+03.3$ & M1-67 & 1.07 & & & & & 682 & & 1000 & $800 \pm 100$ & \\
\hline $051.0+03.0$ & He2-430 & 2.22 & & 2.21 & & & 3972 & & & $>3000$ & \\
\hline $051.0-04.5$ & PC22 & 0.58 & & & & & & & & $4000 \pm 500$ & \\
\hline 051.9-03.8 & M1-73 & 0.88 & & & & 0.95 & 4669 & 4400 & & $2900 \pm 500$ & 4 \\
\hline $052.2-04.0$ & M1-74 & 0.94 & 1.01 & 0.98 & & & 4118 & 8640 & & $5300 \pm 1000$ & \\
\hline $052.5-02.9$ & Me1-1 & 0.05 & 0.72 & 0.46 & & 0.58 & 4618 & & & $<1000$ & 5 \\
\hline $052.9+02.7$ & K3-31 & 2.32 & & & & & 3941 & 6880 & & $800 \pm 700$ & \\
\hline $052.9-02.7$ & K3-41 & 1.13 & & & & & 20464 & 28690 & & $3200 \pm 500$ & \\
\hline $053.3+03.0$ & A59 & & 1.77 & & 1.61 & & 1412 & & 1800 & $<1000$ & \\
\hline $053.8-03.0$ & A63 & 1.12 & & & 1.09 & & & & 2700 & $>8000$ & \\
\hline $055.1-01.8$ & K3-43 & 1.66 & & & & & & & 1500 & $>2800$ & \\
\hline $055.3+02.7$ & He1-1 & 2.06 & & & & & & & & $2500 \pm 1000$ & \\
\hline $055.5-00.5$ & M1-71 & 1.82 & & 1.80 & & 2.06 & & & & $2900 \pm 400$ & 6 \\
\hline $055.6+02.1$ & $\mathrm{He} 1-2$ & 1.86 & & & & & & & & $>3000$ & \\
\hline $056.0+02.0$ & K3-35 & 2.01 & & & & & 3975 & 6580 & & $<1000$ & \\
\hline 057.9-01.5 & He2-447 & 2.18 & & & & & 2874 & & & $>2000$ & \\
\hline $058.9+01.3$ & K3-40 & 1.72 & & & & & 7068 & 6490 & & $>3000$ & \\
\hline $059.0+04.6$ & K3-34 & 0.35 & & & & & & & 5700 & $<1000$ & \\
\hline 059.0-01.7 & $\mathrm{He} 1-3$ & 1.67 & & & & & & & & $1000 \pm 200$ & \\
\hline $059.4+02.3$ & K3-37 & 1.77 & & & & & 7148 & 7910 & & $2400 \pm 400$ & \\
\hline $060.5-00.3$ & K3-45 & 0.84 & & & & & & & 1900 & $<1000$ & \\
\hline $060.5+01.8$ & $\mathrm{He} 2-440$ & 2.94 & & & & & 3962 & & & $>4000$ & \\
\hline $061.3+03.6$ & He2-437 & 1.45 & & & & 0.88 & & & & $1100 \pm 300$ & 7 \\
\hline $062.4-00.2$ & M2-48 & 2.02 & & & & 1.35 & 6970 & & 1600 & $900 \pm 200$ & 8 \\
\hline $067.9-00.2$ & $\mathrm{~K} 3-52$ & 1.49 & & & & & 2459 & 7200 & & $2300 \pm 200$ & \\
\hline $068.6+01.1$ & He1-4 & 1.77 & & & 1.44 & & & & & $>5000$ & \\
\hline $068.7+01.9$ & K4-41 & 1.50 & & & & & 7929 & 7820 & & $>4000$ & \\
\hline $068.7+03.0$ & PC23 & 1.53 & & & & & 6680 & 7740 & & $>4000$ & \\
\hline $068.8-00.0$ & M1-75 & & & & 2.11 & & 3190 & & 3100 & $>4000$ & \\
\hline $069.2+03.8$ & K3-46 & 0.69 & & & & & & & 3200 & $1600 \pm 100$ & \\
\hline 069.6-03.9 & K3-58 & 1.34 & & & & & 5635 & & 5700 & $>3000$ & \\
\hline $071.6-02.3$ & M3-35 & 2.07 & 2.41 & & & & 1760 & 4840 & & $2150 \pm 150$ & \\
\hline $075.6+04.3$ & Anon.20h02 & 0.01 & & & 0.85 & & & & & $7000 \pm 1300$ & \\
\hline $076.3+01.1$ & A69 & & 4.35 & & & & 4173 & & 300 & $>4000$ & \\
\hline $076.4+01.8$ & $\mathrm{KjPn} 3$ & 0.93 & & & & & & & & $2000 \pm 100$ & \\
\hline $077.5+03.7$ & KjPn1 & 1.54 & & & & & & & & $>5500$ & \\
\hline $077.7+03.1$ & KjPn2 & 1.52 & & & & & & & & $3700 \pm 400$ & \\
\hline $078.9+00.7$ & Sd1 & 0.11 & & & & 0.11 & & & & $<1200$ & 9 \\
\hline $084.2-04.2$ & K3-80 & 1.18 & & & & & & & & $900 \pm 100$ & \\
\hline $084.9+04.4$ & A71 & & 1.06 & & & & 722 & & 900 & $2800 \pm 400$ & \\
\hline $088.7+04.6$ & K3-78 & 0.35 & & & & & 7831 & 7290 & & $<1200$ & \\
\hline $089.3-02.2$ & M1-77 & 1.23 & & & & & 5496 & 4880 & 700 & $2000 \pm 250$ & \\
\hline 089.8-00.6 & Sh1-89 & 0.99 & 0.82 & & & & 1941 & & & $1350 \pm 200$ & \\
\hline
\end{tabular}


Table 3. continued.

\begin{tabular}{|c|c|c|c|c|c|c|c|c|c|c|c|}
\hline \multirow[t]{2}{*}{ PNG } & \multirow[t]{2}{*}{ Name } & \multicolumn{5}{|c|}{$c_{\beta}$} & \multicolumn{4}{|c|}{ Distance $[\mathrm{pc}]$} & \multirow[t]{2}{*}{ Reference } \\
\hline & & $\begin{array}{l}\text { Stras. } \\
\text { (1) }\end{array}$ & $\begin{array}{c}\text { Cahn } \\
\text { (2) }\end{array}$ & $\begin{array}{l}\text { Stas. } \\
\text { (3) }\end{array}$ & $\begin{array}{l}\text { this } p \text {. } \\
\text { (4) }\end{array}$ & $\begin{array}{l}\text { Other } \\
(5)\end{array}$ & Cahn & Vst. & Ma. & $\begin{array}{c}\text { THIS } \\
\text { PAPER }\end{array}$ & \\
\hline $091.6-04.8$ & K3-84 & 0.51 & & & & & & & & $5200 \pm 900$ & \\
\hline 093.3-00.9 & K3-82 & 0.96 & & & & & 2675 & 3250 & & $900 \pm 130$ & \\
\hline 093.3-02.4 & M1-79 & 0.13 & 0.78 & 0.77 & & & 2627 & 3610 & 1000 & $4700 \pm 800$ & \\
\hline $093.5+01.4$ & M1-78 & 3.02 & 2.65 & & & & 700 & & 3100 & $>2200$ & \\
\hline $095.1-02.0$ & M2-49 & 1.17 & & & & & 2870 & 6140 & & $>2300$ & \\
\hline $095.2+00.7$ & K3-62 & 2.54 & & & & & 2270 & 4050 & & $>2500$ & \\
\hline $096.3+02.3$ & K3-61 & 1.32 & & & & & 7334 & 6510 & 1100 & $1000 \pm 200$ & \\
\hline 097.6-02.4 & M2-50 & 0.95 & 0.63 & 0.61 & & & 10028 & 9280 & 1500 & $2700 \pm 500$ & \\
\hline $098.1+02.4$ & K3-63 & 1.18 & 0.99 & & & & 5780 & & 1700 & $1400 \pm 130$ & \\
\hline $098.2+04.9$ & K3-60 & 2.27 & 1.90 & 2.59 & & & 3887 & 6210 & & $2000 \pm 200$ & \\
\hline $102.9-02.3$ & A79 & & 1.33 & & 0.81 & 0.36 & 1784 & & 1300 & $1000 \pm 160$ & 7 \\
\hline $103.7+00.4$ & M2-52 & 1.72 & & & 1.44 & & 4411 & & 1500 & $4000 \pm 360$ & \\
\hline 104.4-01.6 & M2-53 & 0.52 & 1.26 & & 1.06 & 0.73 & 3737 & 4940 & 900 & $1900 \pm 400$ & \\
\hline 107.4-00.6 & K4-57 & 1.32 & & & & & & & & $4000 \pm 1000$ & \\
\hline 107.4-02.6 & $\mathrm{K} 3-87^{\dagger}$ & 1.14 & & & & & 9203 & 9680 & & $4400 \pm 800$ & \\
\hline $107.7-02.2$ & M1-80 & 0.52 & & & & & 5496 & 4880 & 1600 & $1500 \pm 200$ & \\
\hline $111.8-02.8$ & $\mathrm{Hb} 12$ & & & 1.35 & & 0.60 & & & & $<1000$ & 11 \\
\hline $112.5-00.1$ & $\mathrm{KjPn} 8$ & 0.67 & & & & 0.42 & & & & $1400 \pm 100$ & 17 \\
\hline $114.0-04.6$ & A82 & & 0.75 & & & & 1868 & & 2000 & $3150 \pm 500$ & \\
\hline $119.3+00.3$ & BV5-1 & 1.05 & & 1.31 & 1.01 & 0.82 & & & & $3000 \pm 400$ & 12 \\
\hline $122.1-04.9$ & $\mathrm{~A} 2$ & 0.76 & 0.56 & 0.34 & 0.80 & & 3929 & & 3000 & $1500 \pm 150$ & \\
\hline $126.3+02.9$ & K3-90 & 0.83 & 0.97 & & & & 5759 & 5600 & & $<1000$ & \\
\hline $128.0-04.1$ & Simeiz22 & & & & & 0.34 & & & & $<1000$ & 13 \\
\hline $130.2+01.3$ & IC1747 & 1.48 & 0.74 & & 1.03 & & 2937 & 2420 & & $1300 \pm 200$ & \\
\hline $130.4+03.1$ & K3-92 & 1.96 & 1.30 & & 1.23 & & 6806 & & & $3100 \pm 900$ & \\
\hline $131.5+02.6$ & A3 & & 1.23 & 1.22 & 1.33 & 1.23 & 2560 & & & $5100 \pm 500$ & 14 \\
\hline $132.4+04.7$ & K3-93 & 1.35 & & & 1.94 & & & & & $<1000$ & \\
\hline $136.1+04.9$ & A6 & & 1.39 & & & 1.39 & 958 & 2240 & & $<1000$ & \\
\hline $138.8+02.8$ & IC289 & 1.03 & 1.09 & & 1.16 & & 1434 & 1480 & & $>8000$ & \\
\hline $142.1+03.4$ & K3-94 & 0.91 & & & & 0.52 & 6507 & 7740 & & $<1000$ & 15 \\
\hline $147.4-02.3$ & M1-4 & 2.47 & 1.84 & 1.62 & & & 2988 & 3390 & & $3300 \pm 350$ & \\
\hline $147.8+04.1$ & M2-2 & 1.34 & 1.50 & 1.50 & & & 4356 & 3880 & & $>2000$ & \\
\hline $151.4+00.5$ & K3-64 & 0.73 & & & & & & & & $1800 \pm 200$ & \\
\hline $153.7-01.4$ & K3-65 & 1.83 & & & & & 10512 & & & $3700 \pm 300$ & \\
\hline $160.5-00.5$ & We1-2 & & & & & 1.44 & & & & $2500 \pm 180$ & 16 \\
\hline $163.1-00.8$ & We1-3 & & & & & 0.70 & & & & $2700 \pm 300$ & 16 \\
\hline $167.0-00.9$ & A8 & & 1.77 & & & 0.68 & 1633 & & & $5500 \pm 1000$ & 2 \\
\hline $170.7+04.6$ & K3-69 & 1.34 & & & & & 7946 & & 17850 & $>6000$ & \\
\hline $178.3-02.5$ & K3-68 & 0.80 & & & & & 5945 & & & $2200 \pm 240$ & 16 \\
\hline $181.5+00.9$ & $\mathrm{Pu} 1$ & & & & & 1.03 & & & & $7000 \pm 800$ & \\
\hline $184.0-02.1$ & M1-5 & & 1.39 & 1.38 & & & 2922 & 4940 & & $>4000$ & \\
\hline $184.6+00.6$ & K3-70 & 1.55 & & & & & 12117 & & & $>6000$ & \\
\hline $184.8+04.4$ & K3-71 & 1.14 & & & & & & & & $2500 \pm 1000$ & \\
\hline $194.2+02.5$ & J900 & 0.56 & 0.64 & 0.63 & & & 2756 & 3270 & & $4300 \pm 650$ & \\
\hline $197.8-03.3$ & A 14 & & 1.18 & & 1.52 & 0.85 & 3317 & & & $5400 \pm 800$ & 10 \\
\hline 201.9-04.6 & We1-4 & & & & & 0.85 & & & & $3000 \pm 1100$ & 15 \\
\hline $204.8-03.5$ & K3-72 & & & & & 1.06 & 5119 & & & $>4000$ & 15 \\
\hline $211.2-03.5$ & M1-6 & 1.84 & 1.17 & 1.83 & & & 2648 & 4290 & & $2000 \pm 160$ & \\
\hline
\end{tabular}

Notes. The NGC objects listed in the Table 1 and the objects listed in Table 2 are omitted in this table. The columns numbered from 1 to 3 give the values of $c_{\beta}$ inferred from $\mathrm{H}_{\alpha}$ and $\mathrm{H}_{\beta}$ supplied to the Strasbourg catalog (1), from Cahn et al. (1992) (2), from Stasińska et al. (1992) (3). The extinctions obtained in this paper are given in Col. 4. In Col. 5, we present the values obtained in other papers, the corresponding references are: (1) van de Steene et al. 1996; (2) Phillips et al. 2005; (3) Girard et al. 2007; (4) Wesson et al. 2005; (5) Shen et al. 2004; (6) Wright et al. 2005; (7) Rodriguéz et al. 2001; (8) López-Martín et al. 2002; (9) Kazarian et al. 1998; (10) Bohigas 2003; (11) Rudy et al. 1993; (12) Bohigas 2008; (13) Kwitter \& Jacoby 1989; (14) Kaler 1983; (15) Bohigas 2001; (16) Kaler et al. 1990; (17) Gonçalves et al. 2009.

The values adopted to obtain the distances are marked in bold-face. If for a PNe no value is shown in bold-face, it means that we find the same results with all of them. Our distance are compared with those obtained by Cahn et al. (1992), van de Steene \& Zijlstra (1994), and Maciel (1984).

${ }^{(\dagger)} \mathrm{K} 3-87$ is a possible symbiotic.

Gaussian. We call the central value of this distribution $d_{A}$ and $\sigma_{d}$ and the dispersion. It follows that $\sigma_{d}=\sigma_{A} / b$.

$d_{A}=\left(A_{\mathrm{v}, i}-a\right) / b$

In Fig. 2, we show as an example the well-defined extinction (4) curve along the line of sight towards NGC 6894. For this nebula, 


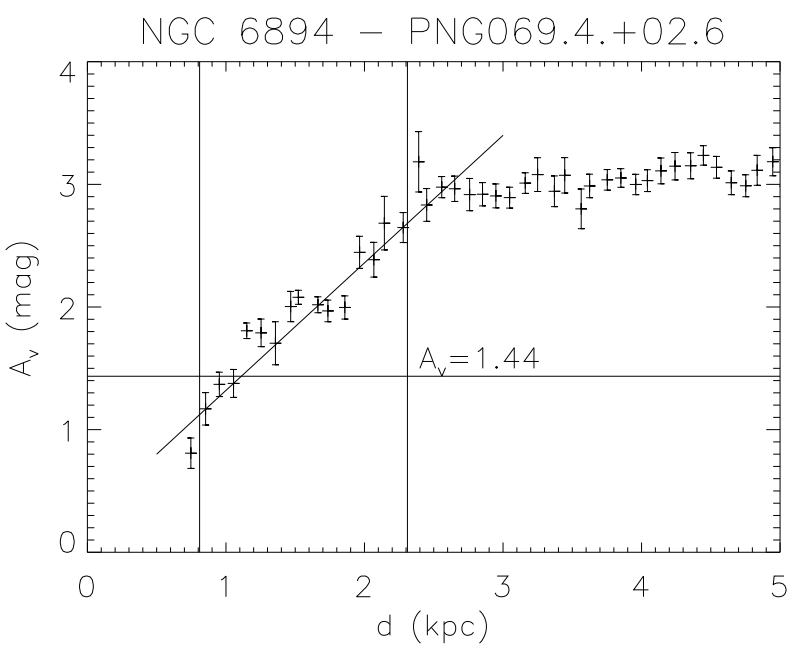

Fig. 2. Extinction law within $10^{\prime} \times 10^{\prime}$ around the line of sight toward the NGC 6894. The measured extinction of the nebula is also indicated by the horizontal line, and the two vertical lines delineate the interval in that the law is linear and that we have used to find the corresponding distance to the PN.

the measured extinction value is $c_{\beta}=0.67$, or $A_{\mathrm{v}}=1.44$ (data from Ciardullo et al. 1999). The limits for the selected linear range are 800 and $2300 \mathrm{pc}$ (sensitivity range of the method). The estimated distance is $d=1000 \mathrm{pc}$ and the formal standard deviation is $\sigma_{d}=150 \mathrm{pc}$. We emphasize that the formal $\sigma$ is only part of the total uncertainty, whose major contribution is due to the error in $c_{\beta}$ measurement; we consider other uncertainties in the following section.

To simplify the discussion in the next section about the uncertainty introduced by the inaccuracies in $A_{\mathrm{v}}$, we fix representative values the parameters $b$ and $\sigma_{d}$. This is achieved by analysing almost two hundred lines of sight. We found a representative mean value for the parameter $b$ of $6.53 \times 10^{-4} \mathrm{mag} / \mathrm{pc}$ (Fig. 3), and a typical error in the fitting process of around $10 \%$. A test distance of $2500 \mathrm{pc}$ is also assumed, which is in the middle of the range over which the extinction-distance method is sensitive for the low Galactic latitude coverage that characterizes the IPHAS survey. This range was calculated by taking the mean of the lower and upper limits of the linear interval corresponding to each curve. For the adopted distance, the typical error in the fitting process is around $250 \mathrm{pc}$. If so, we can express the relative uncertainty in the distance as

$$
E=\frac{\sqrt{250^{2}+\left(\sigma_{A_{\mathrm{v}}} / 6.53 \times 10^{-4}\right)^{2}}}{2500}
$$

$E_{A_{\mathrm{v}}}=\frac{\sigma_{A_{\mathrm{v}}} / 6.53 \times 10^{-4}}{2500}$,

where $E$ is the total uncertainty and $E_{A_{\mathrm{v}}}$ is the contribution of $A_{\mathrm{v}}$. From the previous formulae for $\sigma_{A_{\mathrm{v}}}=0.16\left(\sigma_{c \beta}=0.077\right)$, we obtain $E_{A_{\mathrm{v}}} \sim 10 \%$ and $E \sim 14 \%$. We note finally that the previous formulae are exact in the case of purely statistical errors, otherwise they represent a lower limit to the true uncertainty.

\section{On the errors}

In Sect. 2, we saw how large the difference can be between the $c_{\beta}$ values determined for the same nebula by different authors.

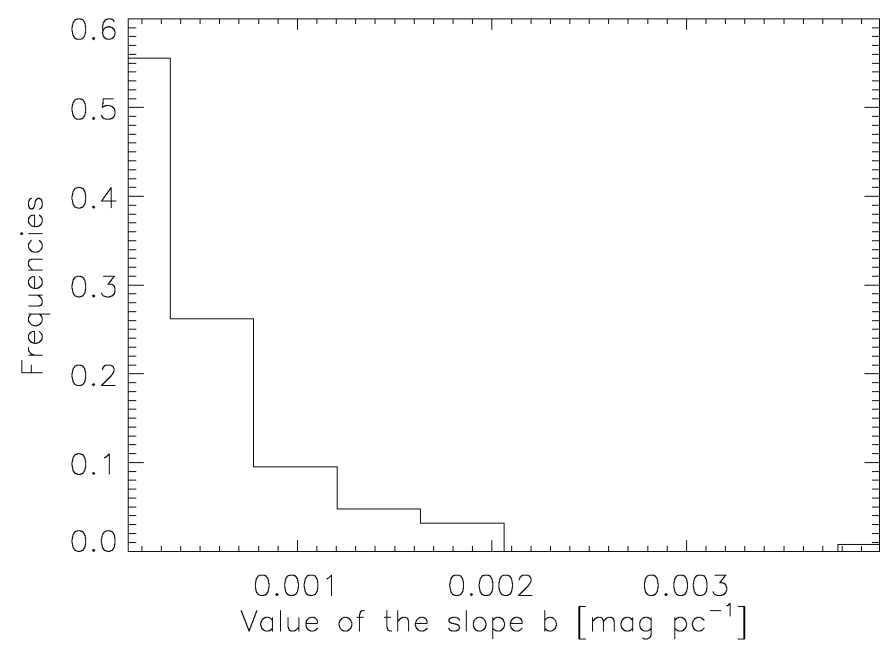

Fig. 3. Distribution of the values of the slope $b \mathrm{mag} / \mathrm{pc}$ obtained from a linear fit of the interstellar extinction curves. We analysed more than 100 lines of sight corresponding to the known PNe in the IPHAS survey. Almost $80 \%$ of the values are distributed between $1.3 \times$ $10^{-4}$ and $8 \times 10^{-4}$.

The origin of these differences could be systematic errors caused by the instruments or the reduction processes. Unfortunately it is impossible to quantify a priori this effect. However, in the determination of the interstellar reddening, hence of the extinctiondistance there are other sources of uncertainty that we discuss below.

\subsection{Sources of error relevant to all objects}

To determine the visual extinction to a $\mathrm{PN}$, it is common to use the $\mathrm{H}_{\alpha} / \mathrm{H}_{\beta}$ ratio. However, the literature data often quote only the extinction coefficient $c_{\beta}$. In some cases, the adopted extinction law is also quoted, in such a way that we can convert the $c_{\beta}$ 's to a common scale, which we assume to be the law given in Fitzpatrick (2004), for $R=3.1$, for a theoretical ratio $\mathrm{H}_{\alpha} / \mathrm{H}_{\beta}=2.86$.

The first uncertainty that we consider is that associated with the theoretical $\mathrm{H}_{\alpha} / \mathrm{H}_{\beta}$ ratio as a function of the electron temperature, for case B. Using Osterbrock (2005) for this ratio, we find that if the electron temperature varies between 8000 and $20000 \mathrm{~K}$, the largest error that we would make by adopting a ratio of 2.86 is 0.04 dex in $c_{\beta}$, or 0.09 mag in visual extinction. This implies an uncertainty of up to $11 \%$ in the distance determination.

The second error source that we consider is the adopted extinction law. Several authors use extinction laws that differ from those adopted in this paper. For example, Stasińska et al. (1992) used the Seaton (1979) law assuming $R=3.2$, Cahn et al. (1992) used the Whitford (1958) law $(R=3.2)$, while the Howarth (1983, $R=3.1$ ) law was used by other authors. The maximum difference between our adopted law occurs when the Cardelli et al. (1989) law is used. We conservatively adopt this difference when the extinction law is not quoted in a paper from which the $c_{\beta}$ for a specific PN is adopted. This results in a difference of $15 \%$ in $c_{\beta}$, or $\sigma_{A_{\mathrm{v}}}=0.3$ (for $A_{\mathrm{v}}=2$ ), and a contribution to the error in the distance of about $20 \%$.

Another common hypothesis is that the characteristics of the interstellar dust are constant for all lines of sight, and correspond to $R=3.1$, the mean value of the Milky Way. 
Fitzpatrick (1999) and Fitzpatrick \& Massa (2007) state that for optical wavelengths a single value of $R$ is a good approximation, but $R$ can vary for different lines of sight. Several authors report $R \sim 2$ through the Galactic bulge (Byun 1996; Udalski 2003; Ruffle et al. 2005) and towards the halo (Larson \& Whittet 2005). Based on the study of a sample of PNe, Stasinska et al. (1992), suggest that for the Galactic plane a more appropriate value is $R=2.5$. The parameter $R$ may instead be significantly larger if the line of sight crosses a dense dust cloud (due to the generally larger size of the grains). However, we would be unlikely to be able to detect the blue part of the spectrum of PNe (and thus their $\mathrm{H}_{\beta}$ flux) along such highly extinguished directions. In general, the $R$ value for a particular nebula can in principle be determined if the number of Balmer lines measured with precision is large enough.

Therefore, we cannot exclude there being significant variations in $R$ for our IPHAS sample. Having adopted $R=3.1$ as a common value, we estimate the error that we make if the true extinction ratio were 2.1 or 5.5 (maximum and minimum values from Fitzpatrick 1999; Fitzpatrick \& Massa 2007). For the case $R=2.1$, the Fitzpatrick law gives $A_{\mathrm{v}}=4.8 \cdot \log \left(\mathrm{H}_{\alpha} / \mathrm{H}_{\beta} / 2.86\right)$, while we use $A_{\mathrm{v}}=6.1 \cdot \log \left(\mathrm{H}_{\alpha} / \mathrm{H}_{\beta} / 2.86\right)$. This results in an overestimate of $A_{\mathrm{v}}$ by some $21 \%$, or $\delta_{A_{\mathrm{v}}}=0.4$ (for $A_{\mathrm{v}}=2$ ). However, in this case the corresponding distance extinction curve is also overestimated by about $15 \%$ (Sect. 3). This means that when determining distances these errors partially compensate and the corresponding overestimate of $A_{\mathrm{v}}$ is about $6 \%$, or about $12 \%$ for a distance of $2500 \mathrm{pc}$. For $R=5.5$, we underestimate $A_{\mathrm{v}}$ by some $33 \%$, but the corresponding extinction curve is also underestimated by $25 \%$, so that a final error is $8 \%$ in $A_{\mathrm{v}}$, or $\sim 14 \%$ for the distance.

\subsection{Uncertainty originating in object properties}

There is additional uncertainty in the hypothesis that the interstellar medium is responsible for all the reddening measured for a PN from the Balmer decrement, in other words, that the PNe are free of internal or circumnebular absorbing dust (for a review see Barlow 1983). This hypothesis is in general supported by the study of Köppen (1977), which, assuming that in a planetary nebula the dust and gas components are well mixed, finds that the nebular dust optical depth is very small $(\tau<0.05)$, for a dust/gas ratio of around $10^{-3}$. However, this could be too simple a picture, and in this original work there were also some PNe (6 out of a total of 21) with evidence of associated extinction. The effect is probably negligible for standard, elliptical PNe (Barlow 1983), but could be more significant for particular nebular morphologies, such as that of bipolar PNe from massive progenitors (e.g. Corradi \& Schwarz 1995), which may have massive neutral equatorial envelopes. There is some evidence in the literature that this might be the case.

The most studied example of the latter type is NGC 7027. Osterbrock (1974) estimated a maximum internal absoption of 0.6 mag. Woodward et al. (1992) suggested that the obscuring dust lies in a shell or disc external to the ionized gas, and Bieging et al. (2008) found variations in $c_{\beta}$ from 0.8 to 2.4. A variation in $A_{\mathrm{v}}$ was also measured for NGC $650-1$ between the SW and NE limits of the central emission bar by Ramos-Larios et al. (2008). Other type-I bipolar nebulae, such as Sh 2-71, K 3-94, $\mathrm{K}$ 4-55, and M 1-75, appear to have some (generally modest) extinction variations across their structures (Bohigas 1994, 2001). Extreme variations are clearly found for NGC 6302 and NGC 6537 (Matsuura et al. 2005a,b).
There are also a few indications of associated extinction for non-bipolar morphologies ${ }^{1}$. In this context, an interesting object is NGC 6741, for which Sabbadin et al. (2005) find a circumnebular neutral halo generated during a recombination phase following the fading of the central star. This halo was estimated to be responsible for $10-20 \%$ of the measured total $c_{\beta}$. The Helix nebula (NGC 7293) seems to be a very complex object in which dust, ionized, and molecular gas cohabit (Speck et al. 2002). Dense knots in a PN could also evolve into absorbing filaments, as reported by O'Dell et al. (2003) for the bipolar PN IC 4406.

For NGC 6781, Mavromatakis et al. (2001) provide a 2D Balmer decrement map, finding variations in $\mathrm{H}_{\alpha} / \mathrm{H}_{\beta}$ ranging from 5.6 to 7 . Though smaller, similar structures are also visible in a similar map obtained for Menzel 1 by Monteiro et al. (2005).

To summarize, in some cases care has to be taken when determining the interstellar extinction, especially for particular geometries (bipolar). However, because of the small number of studies it is difficult to estimate the percentage of objects belonging to these categories whose interstellar extinction could be wrongly estimated. In any case, the number of bipolar PNe is $15 \%$ of the total number of planetary nebulae (Corradi \& Schwarz 1995), and from this subsample significant unrecognized errors are probable only for distant, spatially unresolved objects. We can conclude that only a small fraction of our total sample will be affected by this kind of uncertainty in the estimate.

Concluding, at present the major contribution to the uncertainty in the distances originates in the $A_{\mathrm{v}}$ 's error measurements. For two nebulae, NGC 6842 and NGC 7048, our own measurements allow us to estimate these errors. Combining it with the statistical fitting error leads to a final uncertainty in the distance in about $35 \%$.

\section{Distance determinations for the NGC sample}

To minimize the uncertainties, we infer the distances for a sample of very well known PNe in the IPHAS area, shown in Table 1.

The first PN is NGC 6741 (PNG 033.8-02.6, Fig. 4), which, as discussed in the previous section, was found to have nonnegligible internal extinction by Sabbadin et al. (2005). We use the interstellar value given in the same paper to determine its distance. The reported error in the distance comes from the statistical $\sigma_{d}$. This also applies to the other PNe in this section, except for NGC 6842 and NGC 7048 as discussed later.

For NGC 6781 (PNG 041.8-02.9, Fig. 4) we derive a distance of smaller than $1000 \mathrm{pc}$, by adopting all the reported values of $A_{\mathrm{v}}$, from the minimum value inferred by Mavromatakis et al. (2001) to the maximum value obtained in this paper. We cannot be more precise since the curve is not determined within this range. However, the result is in line with that of Schwarz \& Monteiro (2006), who found a distance of 750 pc using 3D photoionization modeling.

For NGC 6804 (PNG 045.7-04.5, Fig. 4), we use the determination of $A_{\mathrm{v}}$ obtained by Ciardullo et al. (1999), using Hubble images. The graph was transferred to the Fitzpatrick law $(R=3.1)$, which is our adopted law for the interstellar extinction. In this case, the inferred distance is $<800 \mathrm{pc}$, while from our measurement of $c_{\beta}$ and the corresponding error we estimate $850 \pm 70 \mathrm{pc}$. This is a factor of 2 smaller than the statistical distances reported by Cahn et al. (1992) and

\footnotetext{
${ }^{1}$ Note that morphological misclassification my be caused for a fraction of PNe by orientation effects: see Manchado (2004).
} 

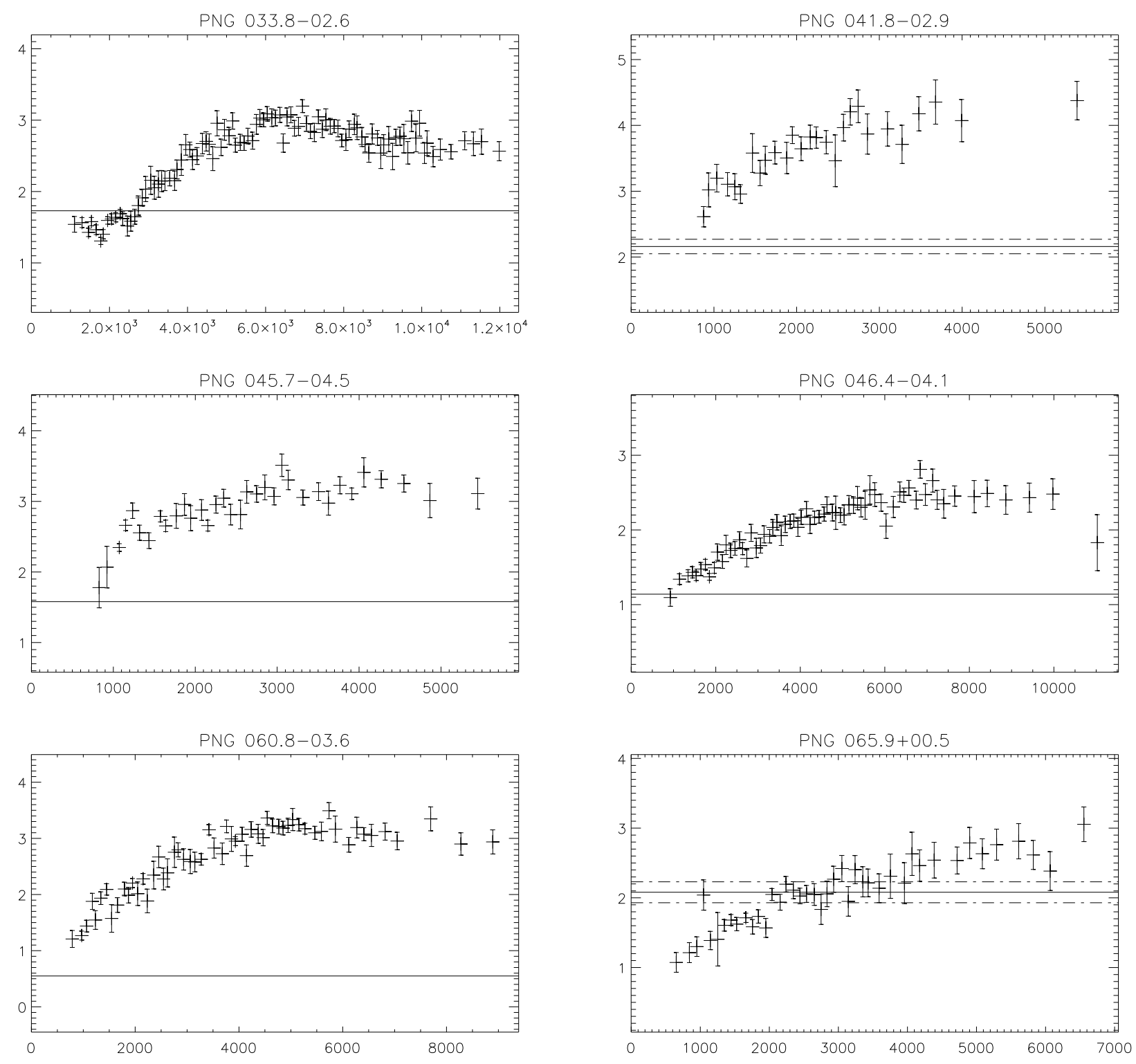

Fig. 4. We present the extinction distance curves for the first 6 nebulae reported in Table 1 . The abscissae are in pc, the ordinates in visual magnitudes, and the horizontal lines represent the visual extinction corresponding to each nebula, as discussed in the text. The short dash-long dash lines represent the $1-\sigma$ error associated with our $A_{v}$ measurement presented in Table 1.

van de Steene \& Zijlstra (1994). The error in the statistical scales is typically $30 \%(1 \sigma)$, so that these values do not exclude the much shorter (and with a smaller uncertainty) distance found here. In support of the smaller distance, we also note that a drawback of the extinction method is a tendency to overestimate distances. Moreover, this is a bipolar nebula for which because of the measured extinction may be significantly higher than the interstellar component because of absorbing structure associated with the PN.

In our method, the only circumstance in which we can underestimate the distance is if the parameter $R>3.1$. This can occur when the line of sight intersects a dense molecular cloud (a rare event). The map of dense molecular clouds in the Galaxy given by Hartmann \& Thaddeus (2001) does not contain any clouds at these coordinates. We therefore recommend the use of our estimated range $<800 \mathrm{pc}$, which is also close to the estimate of 870 pc by Frew (private communication, Frew et al. 2010, in preparation), based on a relationship between size and surface brightness for PNe.
For NGC 6803 (PNG 046.4-04.1, Fig. 4), using the extinction value determined by Peimbert \& Torres-Peimbert (1987) $\left(c_{\beta}=0.53, A_{\mathrm{v}}=1.14\right)$, we infer a distance of $950 \mathrm{pc}$, again smaller than half the previously reported estimates. Even using the much higher values of $c_{\beta}$ presented by Cahn et al. (1992), we obtain smaller distances $(2100 \pm 300 \mathrm{pc})$ than those previously reported.

For the nearby nebula NGC 6853 (PNG 060.8-03.6, Fig. 4), a parallax measurement was performed by Benedict et al. (2003) yielding a distance of $417_{-65}^{+49} \mathrm{pc}$ and a visual extinction coefficient tabulated in the column labeled "Others" of Table 1 . In this case, the nebula is outside of the sensitivity range of our method and we can provide only an upper limit to the distance.

For NGC 6842 (PNG 065.9+00.5, Fig. 4), there is little information in the literature. From our own measurement obtained at the $2.5 \mathrm{~m}$ Isaac Newton Telescope on La Palma, we determine an extinction measurement that is clearly higher than that inferred by adopting line fluxes from the Strasbourg catalog. Observations imply a distance of $2700 \pm 950 \mathrm{pc}$. The error in 

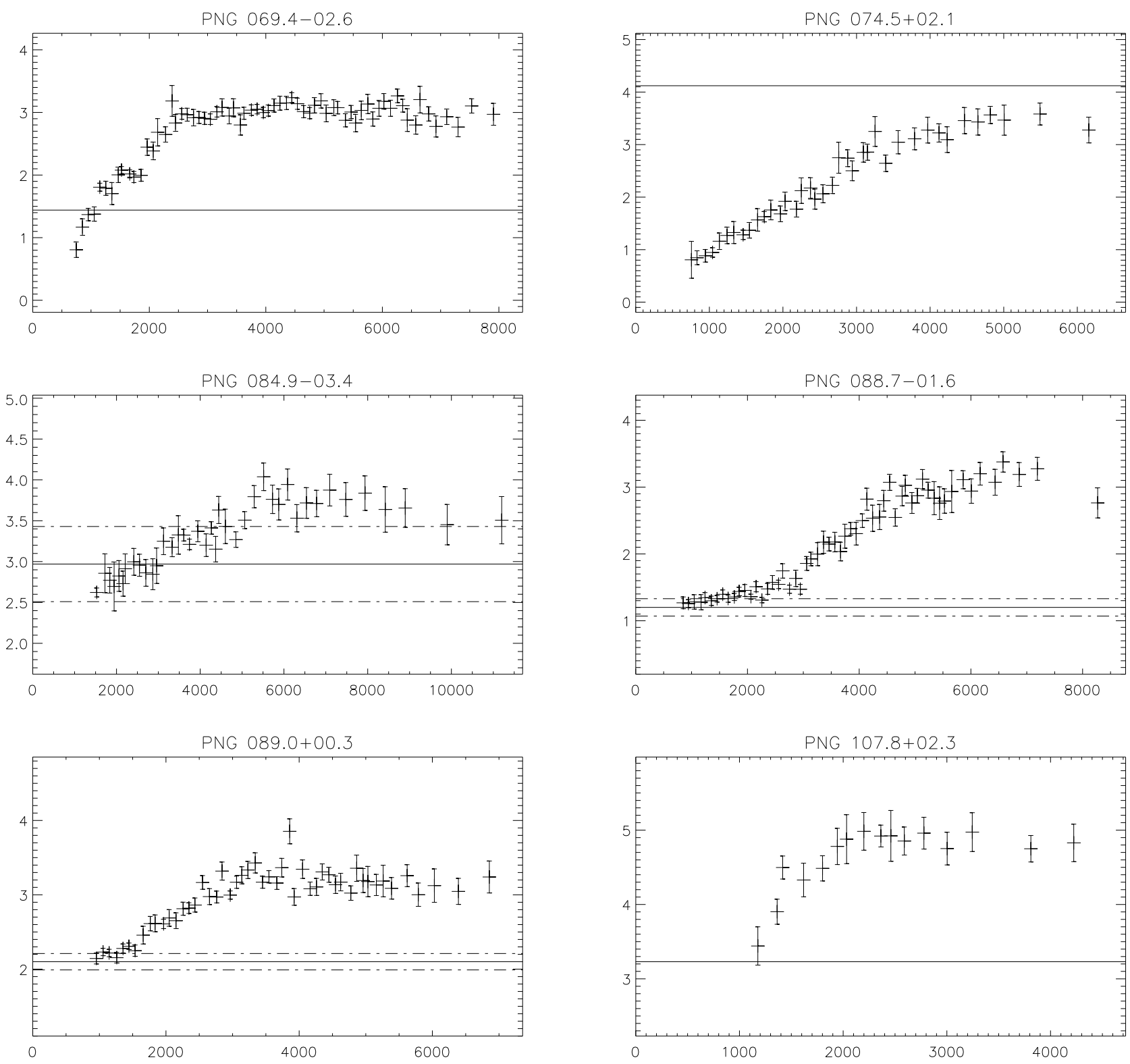

Fig. 5. Extinction-distance curves for the second group of six nebulae presented in Table 1. For all graphics, the abscissae are in pc, the ordinates, in visual magnitudes. The horizontal lines represent the visual extinctions corresponding to the nebulae considered in the text. The short dash-long dash lines represent the 1- $\sigma$ error associated with our $A_{\mathrm{v}}$ measurement presented in Table 1.

the distance has been calculated by taking into account the fitting $\sigma_{d}=749 \mathrm{pc}$, the error in the extinction measurement 0.15 , and the slope of the curve, $b=2.6 \times 10^{-4} \mathrm{pc} / \mathrm{mag}$.

NGC 6881 (PNG 074.5+02.1, Fig. 5) is a quadrupolar young nebula (Guerrero \& Manchado 1998) suggested by Sabbadin et al. (2005) to have been produced by a massive progenitor and presently in a similar recombination phase to NGC 6741. If so, it is possible that $c_{\beta}$ overestimates the interstellar extinction and thus the distance using the extinction method, which would then be greater than $4000 \mathrm{pc}$ using the very high absorption reported in column "Others" (Kaler \& Kwitter 1987), as well as the value obtained from the Strasbourg catalog. For this reason, we do not quote a distance in Table 1.

NGC 6894 (PNG 069.4-02.6, Fig. 5) and NGC 7048 (PNG 088.7-01.6, Fig. 5) are morphologically simpler. The $A_{\mathrm{v}}$ of NGC 6894 is taken from Ciardullo et al. (1999). The extinction coefficient determinations for NGC 7048 are more contradictory. By adopting the lowest one, we found that this is a nearby nebula
$(<1000 \mathrm{pc})$. However, any distance closer than $1000 \mathrm{pc}$ makes the central star absolute magnitude so faint that by using a standard evolutionary track we infer an age of $\gg 10^{5}$ yrs for the PN, so it is probably more distant. Assuming instead the $c_{\beta}$ obtained by our measurement, which agrees with the value in Sabbadin et al. (1987) $\left(A_{\mathrm{v}}=1.27\right)$, we found a distance of between 1000 and $2000 \mathrm{pc}$.

NGC 7027 (PNG 084.9-03.4, Fig. 5) is the clearest case of strong internal absorption, as discussed in Sect. 5. Given the difficulty in estimating its exact amount as a function of the position at which $c_{\beta}$ is measured, no extinction distance is given in Table 1 for this nebula.

NGC 7026 (PNG 089.0+00.3, Fig. 5) is a bipolar nebula with a low extinction coefficient. Using any of the extinction values in Table 1, we found a distance $<1500 \mathrm{pc}$.

Finally, Benetti et al. (2003) propose NGC 7354 (PNG 107.8+02.3, Fig. 5) as a probable recombining nebula in its first phase, as derived from a detailed study of 
NGC 6818. Using the Feibelman (2000) extinction data and extrapolating the extinction-distance curve to low distances, we found a possible distance of about $1000 \mathrm{pc}$.

\section{Determination of distances for whole sample}

We now present distances for 64 other planetary nebulae in the area of sky covered by IPHAS. For an additional 29 objects, only a lower limit to the distance could be obtained. For $17 \mathrm{PNe}$, we found an upper limit, as given in Table 3. We adopt a distance upper limit when the measured extinction to the nebula is smaller than the first point on the corresponding distanceextinction curve. A distance lower limit is defined when the visual extinction to the nebula lies on the plateau of the interstellar extinction curve. For multiple determinations of $c_{\beta}$, the source of the adopted value is reported in bold-face. For upper and lower limits, if no indication is given it means that the same conclusion holds for all tabulated $c_{\beta}$ 's. The errors in the distances given in Table 3 are the fitting errors $\sigma_{d}$.

For the 14 PNe listed in Table 2, we could not estimate the distances because the measured extinction was too far above the interstellar extinction plateau. According to the discussion of Sect. 2.1 and the results presented in Fig. 1, we assume that we know $c_{\beta}$ with the uncertainty $\sigma=0.41$, which is $0.88 \mathrm{mag}$ in $A_{\mathrm{v}}$, for the data extracted from Acker et al. (1992).

We therefore consider all $\mathrm{PNe}$ with a visual extinction more than 0.88 mag higher than the plateau value to be "above" the plateau. Assuming these nebulae to be above the plateau is a zero order hypothesis, because the measured extinctions are not a homogeneous set of measurements and using a common sigma is not rigorous. However, the PNe listed in Table 2 form a sample for future detailed studies, to understand whether the discrepancy with the reddening-distance curve is caused by measurement errors, or if the nebulae have peculiar physical conditions that affect the determination of $c_{\beta}$ (e.g. very high densities that introduce optical thickness effects), whether they are misclassified objects (symbiotic stars, nebulae around young stars, etc.), or whether there is indeed a non-negligible amount of reddening associated with the PNe themselves and not of interstellar origin.

The inferred distances are compared in Fig. 6 with those of Cahn et al. (1992), based on a modified Shklovsky-Daub method, of van de Steene \& Zijlstra (1994), based on a correlation between radio continuum brightness temperature and radius, and of Maciel (1984), based on a mass-radius relationship established from selected electron densities and distances. It is interesting to note that the first two methods seem to provide larger distances than our extinction method, while the latter seems to provide shorter distances. The spread in these graphs is $4.0 \mathrm{kpc}$ (Cahn et al. 1992, 39 objects), 6.6 kpc (van de Steene \& Zijlstra 1994, 23 objects), and $2.5 \mathrm{kpc}$ (Maciel 1984). It therefore seems that the distances found by Maciel are in closer agreement with ours than those from the other authors. However, the small number of objects considered (16 PNe, nine of which are at an estimated distance smaller than $2 \mathrm{kpc}$ ), prevents any conclusion to be drawn about the present sample.

Comparison with other distance scales would be very valuable to help us understand this problem. A detailed comparison with distances determined via the relation between size and $\mathrm{H}_{\alpha}$ surface brightness (Frew et al. 2006; Frew 2008) will be presented in a future paper, after the global photometric calibration of the IPHAS survey becomes available.
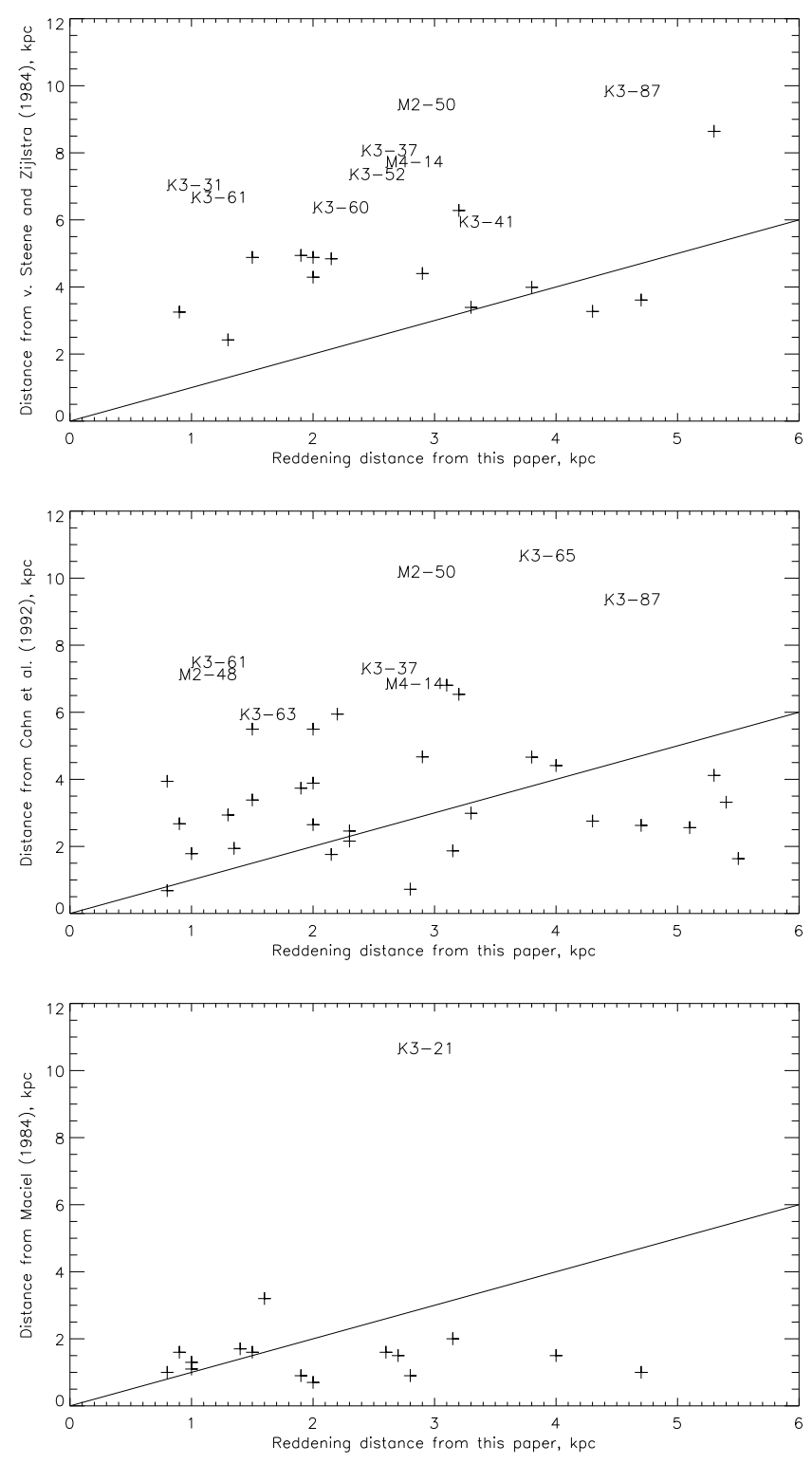

Fig. 6. Comparison between the reddening distance and the statistical determination. The straight line is the 1:1 locus, the statistical distance for the nebulae over the line is greater than that inferred by the extinction. Those PNe for which the difference between the two inferred distances is larger than $4 \mathrm{kpc}$ are labeled in the figure.

\section{Conclusions}

We have discussed the extinction-distance method for determining distances to PNe, motivated by the opportunity provided by the IPHAS survey with its wide application in the Galaxy.

The technique has been presented and the derivation of the distance and its error placed on a formal mathematical basis. We have derived a mean value of the slope $b=6.53 \times 10^{-4} \mathrm{mag} / \mathrm{pc}$ for the galactic extinction distance curve, valid for the analysed region of the sky.

The numerical and physical problems affecting the measurement of the interstellar extinction toward a PN have been discussed. These problems add error to the unavoidable measurement, the effects of an incomplete knowledge of the physics of interstellar dust through the Galaxy and inside PNe, and have to be taken into account every time the method is applied. 
In spite of these uncertainties, which will need to be fully addressed in future, we have computed extinction distances for a sample of $70 \mathrm{PNe}$, which is the largest sample of Galactic PNe to which the technique has been applied. In optimal conditions, when the extinction measurements of the PNe are of good quality and the lines of sight are well behaved in terms of their associated distance-extinction maps, the errors in the PN distances can be as small as $20 \%$, which is a very promising result for a wider application of the method. Unfortunately this is not the common situation for the current data set. As previously mentioned, the uncertainty in the visual extinction for the whole set of nebulae is $\sigma=0.88$, which implies much larger errors in the distances. For a subsample of the PNe considered, extinction is more precisely measured, and for the two cases for which we could determine the associated error, an uncertainty of $35 \%$ in the distance is estimated. This can be taken as a figure to be associated with PNe for which good spectroscopic data exist. A main message of this study is that a careful determination of the nebular extinction is mandatory to limit the errors in such a way that reliable distances can be obtained for individual objects. In this respect, our plan is to improve the extinction measurements for a significant sample of Galactic PNe.

We have compared these new extinction distances with other statistical methods. Some of the methods considered (Cahn et al. 1992; and van de Steene \& Zijlstra 1994) provide distances that are generally larger than our determinations, and in general the spread between our measurements and the statistical determinations is quite high. This emphasizes the large 1- $\sigma$ dispersion in the statistical distances, and the dangers in relying on the determination of a statistical distance to a PN without considering the particular characteristics of a given nebula.

We conclude, by highlighting that the analysis in this paper demonstrates the improved potential of the extinction method provided by the availability of surveys such IPHAS, which provide precise photometric data across large areas of sky. This opens the possibility of making a significant step forward in the calibration of the PN distance scale in the Galaxy, and in our understanding of important issues such as the properties of the dust distribution within the Galactic disc, and the possible importance of dust associated with PNe.

Acknowledgements. C.G., R.L.M.C., A.M., and M.S.G. acknowledge funding from the Spanish AYA2007-66804 grant.

\section{References}

Acker, A. 1978, A\&AS, 33, 367

Acker, A., \& Stenholm, B. 1987, ESO Messenger, 48, 16

Acker, A., Marcout, J., Ochsenbein, F., Stenholm, B., \& Tylenda, R. 1992, (SECGPN) Strasbourg - ESO catalogue of galactic planetary nebulae, Garching: European Southern Observatory

Barlow, M. 1983, Proc. IAU Symp., 103, 105

Benedict, G. F., McArthur, B. E., Fredrick, L. W., et al. 2003, ApJ, 126, 2549

Bensby, T., \& Lundström, I. 2001, A\&A, 374, 599

Benetti, S., Cappellaro, E., Ragazzoni, R., Sabbadin, F., \& Turatto, M. 2003, A\&A, 400, 161

Bieging, H., Boley, P. A., Latter, W. B., \& Tielens, A. G. G. M. 2008, ApJ, 676, 390

Bohigas, J. 1994, A\&A, 288, 617

Bohigas, J. 2001, RMxAA, 37, 237

Bohigas, J. 2003, RMxAA, 39, 149

Bohigas, J. 2008, ApJ, 674, 954

Byun, Y.-I. 1996, ChJPh, 34, 1113

Cahn, J. H., Kaler, J. B., \& Stanghellini, L. 1992, A\&AS, 94, 399

Cardelli, J. A., Clayton, G. C., \& Mathis, J. S. 1989, ApJ, 345, 245
Christianto, H., \& Seaquist, E. R. 1998, AJ, 115, 2466

Ciardullo, R., Bond, H. E., Sipior, M. S., et al. 1999, AJ, 118, 488

Corradi, R. M. L., \& Schwarz, H. E. 1995, A\&A, 293, 871

Cuesta, L., Phillips, J. P., \& Mampaso, A. 1996, A\&A, 313, 243

Drew, J. E., Greimel, R., Irwin, M. J., et al. 2005, MNRAS, 362, 753

Feibelman, W. 2000, PASP, 112, 861

Fitzpatrick, E. L. 1999, ASP, 63, 75

Fitzpatrick, E. L. 2004, ASPC, 309, 33

Fitzpatrick, E. L., \& Massa, D. 2007, ApJ, 663, 320

Frew, D. J. 2008, unpublished Ph.D. Thesis, Macquarie University

Frew, D. J., Parker, Q. A., \& Russeil, D. 2006, MNRAS, 372, 1081

Gathier, R., Pottasch, S. R., \& Pel, J. W. 1986, A\&A, 157, 171

Girard, P., Köppen, J., \& Acker, A. 2007, A\&A, 463, 265

Gonçalves, D. R., Mampaso, A., Corradi, R. L. M., \& Quireza, C. 2009, MNRAS, 398, 2166

Guerrero, M. A., \& Manchado, A. 1998, ApJ, 508

Hartmann, D. T. M., \& Thaddeus, P. 2001, ApJ, 547, 792

Howarth, I. D. 1983, MNRAS, 203, 301

Kaler, J. B. 1983, ApJ, 271, 188

Kaler, J. B., \& Kwitter, K. B. 1987, PASP, 99, 952

Kaler, J. B., Shaw, R. A., \& Kwitter, K. B. 1990, ApJ, 359, 392

Keler, J. B., Kwitter, K. B., Shaw, R. A., \& Browing, L. 1996, PASP, 108, 980

Kazarian, M. A., Parsamian, S., \& Parrao, L. 1998, Astrophys., 41, 239

Köppen, J. 1977, 56, 189

Kwitter, K. B., \& Jacoby, G. H. 1989, AJ, 98, 2159

Larson, K. A., \& Whittet, D. C. B. 2005, AJ, 623, 897

López-Martín, L., López, A., Esteban, C., et al. 2002, A\&A, 388, 652

Lutz, J. H. 1973, ApJ, 181, 135

Maciel, W. J. 1984, A\&AS, 55, 253

Manchado, A. 2004, ASPC, 313, 3

Manchado, A., Riera, A., Mampaso, A., García Lario, P., \& Pottasch, S. R. 1989 RMxAA, 18, 182

Matsuura, M., Zijlstra, A. A., Gray, M. D., Molster, F. J., \& Waters, L. B. F. M. 2005a, MNRAS, 363, 628

Matsuura, M., Zijlstra, A. A., Molster, F. J., et al. 2005b, MNRAS, 359, 383

Mavromatakis, F., Papamastorakis, J., \& Paleologou, E. V. 2001, A\&A, 374, 280

Monteiro, H., Schwarz, H. E., Gruenwald, R., Guenthner, K., \& Heathcote, S. R. 2005, ApJ, 620, 321

O’Dell, C. R., Balick, B., Hajiam, A. R., Henney, W. J., \& Burkert, A. 2003, RMxAA Ser. Conf., 15, 29

Osterbrock, D. E. 1974, PASP, 86, 609

Osterbrock, D. E. 2005, Astrophysics of gaseous nebulae and active galactic nuclei (University Science Books), 73

Peimbert, M., \& Torres-Peimbert, S. 1987, RMxAA, 15, 117

Peña, M. 2005, RMxAA, 41, 423

Peña, M., Stasińska, G., \& Medina, S. 2001, A\&A, 367, 983

Phillips, J. P., Cuesta, L., \& Kemp, S. N. 2005, MNRAS, 357, 548

Pollacco, D. L., \& Ramsay, G. 1992, MNRAS, 254, 228

Ramos-Larios, G., Phillips, J. P., \& Cuesta, L. 2008, MNRAS, 391, 52

Rodgers, A. W., Conroy, P., \& Bloxham, G. 1988, PASP, 100, 626

Rodriguéz, M., Corradi, R. L. M., \& Mampaso, A. 2001, A\&A, 377, 1042

Rudy, R. J., Rossano, G. S., Erwin, P., Puetter, R. C., \& Feibelman, W. A. 1993, AJ, 105, 1002

Ruffle, P. M. E., Zijlstra, A. A., Walsh, J. R., et al. 2004, MNRAS, 353, 796

Sabbadin, F., Cappellaro, E., \& Turatto, M. 1987, A\&A, 182, 305

Sabbadin, F., Benetti, S., Cappellaro, E., Ragazzoni, R., \& Turatto, M. 2005, A\&A, 436, 459

Sale, S. E., Drew, J. E., Unruh, Y. C., et al. 2009, MNRAS, 392, 497

Schwarz, H. E., \& Monteiro, H. 2006, ApJ, 648, 430

Seaton, M. J. 1979, MNRAS, 187, 73

Shen, Z.-X., Liu, X.-W., \& Danziger, I. J. 2004, A\&A, 422, 563

Speck, A. K., Meixner, M., Fong, D., et al. 2002, AJ, 123, 346

Stasińska, G., Tylenda, R., Acker, A., \& Stenholm, B. 1992, A\&A, 266, 486

Stenholm, B., \& Acker, A. 1987, Proc. Frascati Workshop Planetary and Protoplanetary Nebulae from IRAS to ISO, ed. Praite-Martínez, 25

Tylenda, R., Acker, A., Stenholm, B., \& Köppen, J. 1992, A\&AS, 95, 337

Udalski, A. 2003, ApJ, 590, 284

van de Steene, G. V., \& Zijlstra, A. A. 1994, A\&A, 108, 485

van de Steene, G. C., Jacoby, G. H., \& Pottasch, S. R. 1996, A\&AS, 118, 243

Warner, J. W., \& Rubin, V. C. 1975, ApJ, 198, 593

Wesson, R., Liu, X.-W., \& Barlow, M. J. 2005, MNRAS, 362, 424

Whitford, A. E. 1958, AJ, 63, 201

Wright, A., Corradi, R. L. M., \& Perinotto, M. 2005, A\&A, 436, 967

Woodward, C. E., Pipher, J. L., Forrest, W. J., Moneti, A., \& Shure, M. A. 1992, ApJ, 385, 567 